\title{
Research on Transplanted Design of Mobile-Terminal-Based Educational Games
}

\author{
Yan Gu, Na Tian \\ Department of Educational Technology, Jiangnan University, Wuxi, China \\ Email:zero gy@163.com
}

Received January 2014

\begin{abstract}
The number of educational games developed in mobile terminals becomes more and more. The types can be divided into transplanted development and innovative development. Transplanted design is based on existed educational games, and the games are redesigned into mobile terminals, but they have problems about poor user experience and too many similarities between them. This paper analyses the causes of these phenomena and gives some suggestions. According to games from personal computers, their interaction styles should be redesigned.
\end{abstract}

\section{Keywords}

Mobile Terminals, Education Games, Transplanted Design

\section{Introduction}

As the wave of mobile information technology sweeping the world, mobile communication terminals (e.g. Smart Phones, Pads) are gradually replacing personal computers. Android and iOS are becoming dominated operating systems, and people use these applications developed on them more and more often. As a result, more and more companies and designers focus on the design and development of mobile applications (APP), in which, educational APPs become central issue. Take iOS for example, the total number of APPs in App Store is almost $1,000,000$ up to the end of 2013, in which educational APPs take $11 \%$ and rank second following the games APPs. At the same time, the income from educational APPs ranked fourth in the whole year all over the world. These cases demonstrate that mobile terminals are becoming new educational platform.

Educational games take the large share of educational APPs. Take one day in December 2013 for example, the number of educational games in the top paid 100 educational APPs is 83. This demonstrates that the number is large, people would like to pay for them, and the market demand for educational games will be larger. But the existed educational games have problems about too many similarities between them and developers gain market share by copying. As a result, many educational games made in China face several problems, such as low user satisfaction, low open frequency, short staying time in terminals, and then will be eliminated by the market at the end. In order to change this current situation, we have to improve the quality of the educational games and enhance the game attraction.

Currently, mobile terminal based virtual education games development are divided into two classes, one is innovative development, in which both style and content of the game are new and developed according to new 
platform; the other is transplanted development, in which the games are transplanted from real games or PC games, and the styles, contents or rules are modified according to the device property to be suitable for the new platform.

Several Chinese researchers have been focusing on researching of educational games. Wei Ting [1] investigated educational games from views of design idea, design method and structure design. Song Minzhu [2] proposed EFM, a model for educational game design. Shang Junjie [3] has done empirical study about Edutainment game. However, the above researches have never realized the difference between innovative development and transplanted development. Through the early transplanted game design, several developers with experience have been trained, and the game design rules have been established. Therefore, the cost of development is low and it is easy to spread. While innovative design requires new game model, rules and scene. Users need additional learning about the new rules and operating styles. But the present situation is that the successful APPs in App Store are innovative, while the transplanted APPs occupy a large share. If a better solution can be found to solve the problems in transplanted APPs, it will improve the quality of a large part of educational games.

\section{Problems}

\subsection{Poor User Experiences}

Influenced by original game styles, design thinking of transplanted game is relatively fixed. Some games adopt virtual button to replace keyboard operation. However, lack of a raised button makes lower handling operation. Some games adopt touch screen operation to replace mouse, but the accuracy is lower than the mouse and the operator's own finger interference make poorer user experience.

While the innovative APPs are able to develop games according to the own feature of device, for example, there are a lot of interaction styles for mobile communication terminals, we can obtain program response by multipoint touch, voice input, gravity sense, video input, GPS etc. One educational game named Play Science, as Figure 1 shows, makes ultimate use of these features, design games by using various interaction styles efficiently. It simulates generating heat by repeating friction with the touch screen, simulates blowing cool by blowing into microphone, and simulates effects of inertia on objects by shaking the phone.

\subsection{Serious Similarities between Games}

Both the real games and games developed in PC are famous to some degree. For different developers, it is easy to train users and does not exist copyright problems, they all enter the transplant educational game area. As a result, the contents and styles of the games have great similarities, and often lose user loyalty. For example, chess as a puzzle game is popular for thousands of years, has a great number of users. User experience of this game is almost the same except some rules details. Therefore, there is little difference between all versions of chess games. It is great challenge for developers to give different user experience in so large number of similar APPs.

\section{Analysis}

\subsection{Neglecting the Original User Experiences}

Transplanted educational game is difficult to succeed, and one of the important reasons is neglecting user experience. For the happy experience in the original games, users choose to play these similar games in mobile terminals. But the actual impression is beyond the expectation, so the comments are often negative. Actually, it is difficult to gain good user experience if educational games are directly transplanted into mobile platforms.

Firstly, it is difficult to gain similar game experience. The original existed educational games have been gradually modified and improved, made good balance between education and entertainment. Besides, the game calls the comprehensive ability of the participants. Therefore, it is a difficult task to transplant the traditional real games to mobile terminals. Take Pick Sticks for example, players need to throw the sticks randomly, and then pick the sticks one by one without touching another stick. This game is helpful for training the ability of observation, finger force control and strategizing. However, when players play this game transplanted in mobile terminals, they will find the game easy and what they need to do is to click the top stick. 


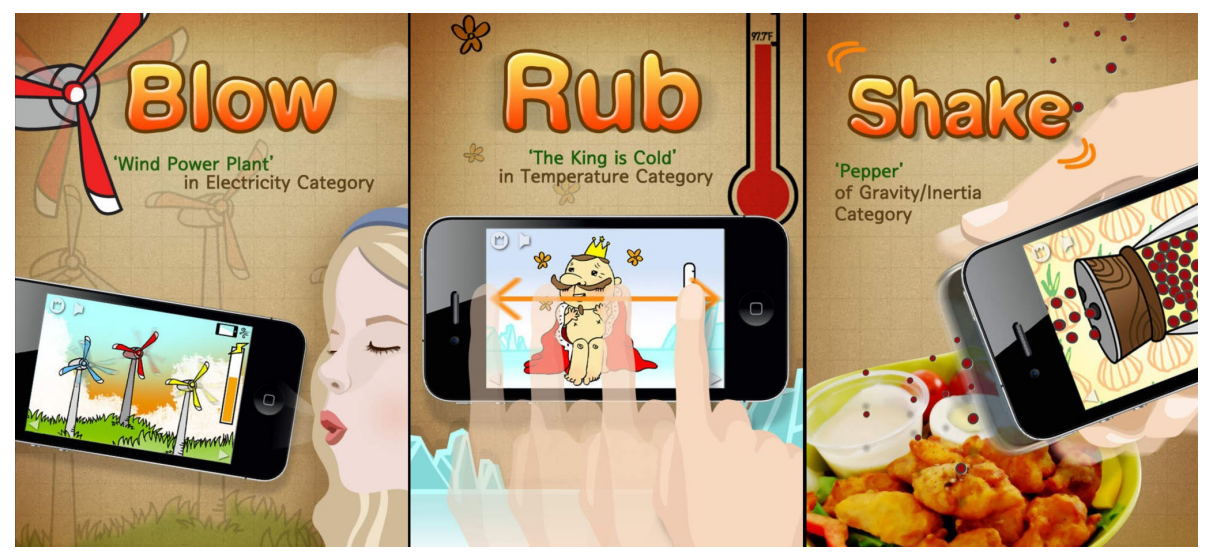

Figure 1. The game named Play Science.

Therefore, the player who is the fastest will win the game. While these experiences in real games, such as the training of muscles to control fine action, the multi-degree observation to find the right stick and the strategy to throw sticks to minimize the difficulty of the game, cannot be obtained in the transplanted game.

Secondly, it is hard to get firm game partners. Participants in real games are living, and they can confirm each other, usually have had real relationship. Participants in PC games spend much time on them, and can also get relatively firm Internet game partners. These close relationships between game partners can improve the attraction of game. Players would like to spend more energy and time on games. It is also relatively easy to adjust the game rules because of such relationships. Therefore, games will develop sustainable and variable. However, fragmentation, the special property of mobile terminals, result in that heavy users and emotional relationship are hard to be cultivated.

Finally, transaction in the application market results in that developers are difficult to understand the requirements of real users. For example, in App Store, users are required to register and bind the credit card or other payment methods, they also need to be confirmed again when downloading APPs. Adults, not minors (the real users of educational games APPs) are registering and downloading APPs. Therefore, the number of downloading demonstrates the requirements of parents not the real users. Developers will go to the wrong direction if they pay too much attention on the number. For example, parents prefer educative to game-play, while children (the real players) prefer game with more entertainment. If developers pay too much attention on the requirements of parents and prefer educative to game-play in the designing process, the developed APPs cannot keep children's interest and cannot reach the purpose of learning through game, even they can get parents' approve and large downloads.

\subsection{Neglecting Special Feature of the New Platform}

Mobile terminals generally have the following features: mobility, which makes Mobile Learning feasible; more interaction, which means diverse and fast interactive experience; fragmentation, which means that the attention can be easily interfered.

Firstly, processes of the game are not synchronized on different platforms. Some games can be run on different platforms, such as PC, smart phone, pad. But users cannot continue the game on one platform if they stop on another platform, which result in reduction of the willingness on the game.

Secondly, single interactive style. In real educational games, body action or voice can be used response which makes the game continue. Take Word Solitaire for example, one player speaks one word whose first letter is the last letter of the word spoken by previous player. This game chooses voice as the interactive styles. Educational games on PC adopt mouse, keyboard, microphone, and camera as the input devices to drive the game. In design of the transplanted games, interaction styles have to be improved to obtain good game experience.

Finally, personalized experience is missing. Mobile terminals reflect strong personal color, such as location, frequency of using APPs, and cookies which can exactly work out users' attention, interest, etc. In good educational games, the behaviors of users reflect the mastery of knowledge, according which the difficulty 
can be adjusted. While most of the educational games APPs cannot achieve that.

\section{Solutions}

\subsection{Transplanted Design of Educational Games on PC}

Educational games on PC are building a virtual game space like mobile terminals, whose contents are suitable for transplanting. The difficulty for transplanting is to redesign the interactive styles which base on mobile terminals.

Virtual buttons should be carefully used and can be replaced by gesture. For example, the popular pinch gesture zooming out the pictures replace the mouse wheel. If the real buttons in keyboard are used as shortcuts in games, it is bad solution to use virtual button on mobile terminals to replace. For the soft surface of the touch screen, the virtual button should be observed by eyes not only by fingers, which result in reduction of the game-play. Take the Parkour for example, the keys of direction, numbers are used to control actions of up, forward, back on PC. While on mobile terminals, users only need to click to control jumping up and the rest actions can be done automatically, which ensures fluent experience.

Diverse interactive styles enhance operating fun. For example, one educational game about constellation knowledge named as Star Walk, as Figure 2 shows, makes use of cameras and GPS. When users take the cameras towards the sky, the constellation diagram and the sky can be showed on the real-time screen. Users can easily know which constellation the stars belong to. The new functions of the mobile terminals extend the types of the virtual educational games, which enhance the educative and game-play by applying properly.

\subsection{Transplanted Design of Real Educational Games}

Virtual educational games are different from real educational games. In the transplanted process, virtual educational games are just treated as an assisted educational tool, and cannot replace real games. Take Ocarina2 for example, microphone and screen are set as response devices. Users simulate playing flute by blowing to microphone and press fingers on different holes. This APP can cultivate the interest of playing instruments, but cannot replace the real learning process. Therefore, we should pay attention to the following points:

Firstly, when choosing the transplanted educational games, we should avoid games with too much focus on body actions [4], especially for large muscle training. For example, Ball games adopt gyroscopes in mobile devices with gravity sense function, which is helpful for training small muscles and fine actions. When transplanting into mobile terminals, puzzle educational games have some superiorities and even are able to obtain better performance of educative and game-play. For example, a bridge building game named as Bridge Constructor Playground, as Figure 3 shows, the building style is the same as real building with blocks. For the superiority of mobile terminals, the bridge can be built with dragging steels, woods, concretes and wire cables. The complete bridge can be tested by vehicles. Furthermore, the load bearing of the bridge is also required to be improved within the budget. Through constantly practicing, structure knowledge and plan ability of the user are able to be improved.

Secondly, basing on the real educational games, games can be extended according to the device properties, in order to form differentiation superiority among APPs with the same type. For example, one version of Ball game not only adopts traditional balls, but also sets the route of balls as maze, in which, lights are only around the balls. Another version of Ball game named as Blendamaze is incorporated with knowledge of colors. When the balls pass different color blocks, they will be colorized with various colors and reach the purpose of color effects of destination. Design of the two ball games enables traditional ball game increase multi-game experience. Take Smart Go Player for example, multiple settings are provided to users. Users can choose real double players or play with the machine. Besides, the machine will adjust the handicap number according to the wining probability. The game, provided with tutorials, can be played as a learning assisted tool and has obvious superiority over other simple chess games.

Finally, due to the inherent fragmentation characteristics of mobile learning, special attention needs to be paid on the game design. Single-stage learning time should not be too long, and some games may be interspersed during the education process. For example, an APP named as Playful Minds: Math, carefully decomposes the learning contents of mathematics, and each stage only has 10 subjects. There will be a small game after completing 5 stages. During the process, golden coins are saved and can be used to buy clothes to dress up players. 


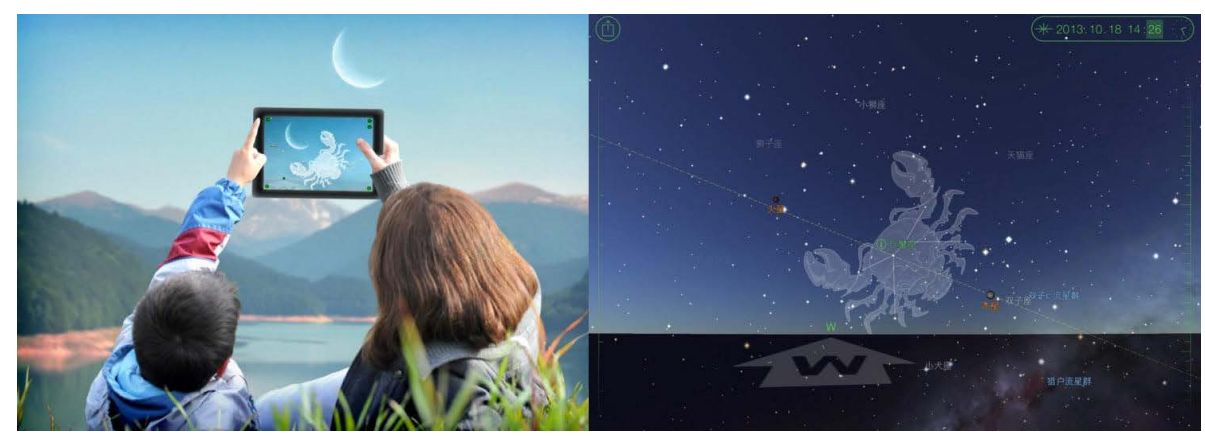

Figure 2. The game named Star Walk.

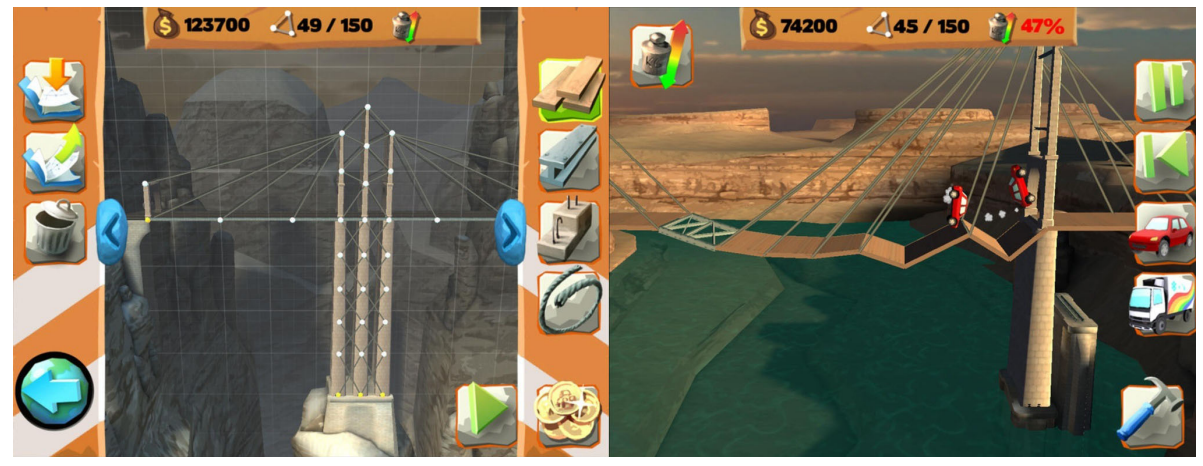

Figure 3. The game named Bridge Constructor Playground.

These control designs of game processes limit the length of the learning time unit, and achieve a better balance of educative and game-play of the whole educational game.

\section{Conclusion}

The new features of mobile terminals, such as portability, diversity, usability, are becoming a beneficial platform for educational games. Transplanted design of various educational games to mobile terminals can not only enrich educational resources, cultivate learning interest, enhance various accomplishments, but also easy to learn and play due to the low cost of development. During the design process, the original games have to be redesigned, such as adjusting operation styles, innovative contents, fragmentation teaching, improving entertainment, etc. Therefore, optimizing the operation experience, and obtaining balance between educative and entertainment, the transplanted design will succeed basing on the original games.

\section{References}

[1] Wei, T. and Li, Y. (2009) Summary of Educational Games Design Research. Journal of Distance Education, 3, 67-70.

[2] Zhu, M.Z. and Zhang, S.J. (2009) EFM: A Model for Educational Game Design. China Educational Technology, 1, 24-27.

[3] Shang, J.J., Li, F.L. and Li, H.W. (2005) Edutainment Is the New Field of Educational Technology. Journal of Distance Education, 1, 24-26.

[4] Gardner, H. (2008) Frames of Mind: The Theory of Multiple Intelligence. 5th Edition, China Remin University Press, Beijing. 